\title{
Impact on mental health of the COVID-19 pandemic in a radiation oncology department
}

\author{
Morena Caliandro $^{1}$ - Gregucci Fabiana ${ }^{1} \cdot$ Alessia Surgo $^{1}{ }^{10} \cdot$ Roberta Carbonara $^{1} \cdot$ Maria Paola Ciliberti $^{1}$. \\ Ilaria Bonaparte ${ }^{1} \cdot$ Sergio Caputo $^{2} \cdot$ Alba Fiorentino $^{1}$
}

Received: 13 September 2021 / Accepted: 3 December 2021 / Published online: 16 January 2022

(c) Italian Society of Medical Radiology 2022

\begin{abstract}
Aim To evaluate the emotional state and organizational well-being of healthcare workers in radiation oncology departments, during the COVID pandemic.

Methods A survey was carried out with three questionnaires: Impact of Event Scale-Revised (IES-R); Italian ANAC questionnaire; and Maslach Burnout Inventory (MBI). Comparisons between groups were done by Student's $t$ test.

Results Seventy-eight questionnaires for 26 workers were analyzed. Thirty-three percent of the sample obtained an IES-R high score, such as post-traumatic syndrome. In terms of organizational well-being, younger age and lower working seniority were statistically significant for higher score of ANAC items $(p<0.5)$. Regarding MBI, 0,27 and 50\% high scores of emotional exhaustion, depersonalization and personal accomplishment were reported, respectively. Low working seniority and male sex were correlated with high score of personal accomplishment ( $p: 0.05 ; p: 0.03)$.

Conclusion Intervention to promote mental health well-being should be implemented in radiation oncology department.
\end{abstract}

Keywords Radiotherapy $\cdot$ COVID $\cdot$ Burnout $\cdot$ Stress

\section{Introduction}

In December 2019, a cluster of pneumonia cases was identified as the SARS-CoV-2 or COVID-19 [1]. On March 2020, the World Health Organization declared the COVID-19 as a pandemic. In the present context, the COVID-19 infection had a significant impact on everyone's daily life; in fact, emerging evidence in general population suggests that the fear of infection is associated with higher levels of perceived stress [2].

A recent meta-analysis, focused on the prevalence of COVID-19 psychological symptom era, showed that $29.6 \%$ of the general population reported high levels of stress, $31.9 \%$ reported anxiety and $33.7 \%$ reported depression [3].

Alessia Surgo

surgo.alessia@gmail.com

1 Radiation Oncology Department, General Regional Hospital F. Miulli, Strada Provinciale 127, 70020 Acquaviva delle fonti (BA), Italy

2 General Regional Hospital F. Miulli, Acquaviva delle fonti (BA), Italy
Furthermore, the life of healthcare workers received additional stress with psychological symptoms including anxiety, stress and sleep disturbances [4-10] during the pandemic. Several studies have been conducted on COVID-19 physicians, urologists, pharmacists and general practitioners [4-12].

Treating oncological patients increased the risk of burnout syndrome as reported in several studies and meta-analysis in radiotherapists and radiation oncologists [13-15]. To our knowledge, no data in terms of psychological quality of life were reported on oncological workers, especially in a radiation (RT) oncology department.

Thus, based on this background, the aim of the present analysis was to evaluate the emotional state and organizational well-being of healthcare workers in an oncological department, especially in radiation oncology, during the COVID-19 pandemic. 


\section{Methods}

A survey was carried out on April-May 2021, 1 year after the first lockdown in Italy, with the aim to assess the impact of COVID-19 on the mental health of Radiation Oncology Department workers.

Three psychological validated self-report questionnaires were used:

(a) To evaluate post-traumatic stress symptoms, the Impact of Event Scale—Revised (IES-R) was used [16]. Significant symptoms were defined by a score more than 33 ;

(b) To evaluate the organizational well-being, the Italian ANAC questionnaire was used. It was useful to detect the opinions of employees with respect to the organization and the working environment; identifying, consequently, possible actions to improve the general conditions [17]. The questionnaire is structured in three sections: 1) Organizational well-being (questions from A to I);2) Degree of sharing of the evaluation system (questions from L to N); 3) Evaluation of the hierarchical superior (Questions $\mathrm{O}$ and $\mathrm{P}$ ). High scores defined a well organization;

(c) To evaluate emotional exhaustion (EE), depersonalization (DP) and personal accomplishment (PA), the Maslach Burnout Inventory (MBI) was utilized [18]. EE evaluated the feeling of exhaustion, DP measures a cold and impersonal response toward the patients, PA evaluated the feeling of one's competence and desire for success. Higher scores $\geq 30$ and $\geq 12$, respectively, for EE and DP and lower scores inferior to 33 for PA, defined high burnout symptoms.

\section{Statistical analysis}

Data were expressed as means and standard deviations (SD) or percentage. Comparisons between groups (gender, age, working seniority) were done by Student's $t$ test or chi-2 test, where appropriate, using SPSS ver.13.

\section{Results}

The response rate was $100 \%$. Seventy-eight questionnaires with 178 questions were analyzed. The sample consists of 26 workers: five radiation oncologists, three medical physicists, one psych-oncologist, 3 nurses, 9 radiotherapists, 4 workers of administrative staff and one site coordinator.
Table 1 Characteristics of sample

\begin{tabular}{lll}
\hline & $N$ & $\%$ \\
\hline Women & 19 & 73 \\
Men & 7 & 27 \\
Working seniority inferior to 5 yo & 20 & 77 \\
Working seniority superior to 5 yo & 6 & 23 \\
Age inferior to 30 & 11 & 42.3 \\
Age superior to 30 & 15 & 57.7 \\
Managerial role & 9 & 34.6 \\
No managerial role & 17 & 65.4 \\
\hline
\end{tabular}

$N$ number of participants; $\%$ percentage

Median age was 32 years (range 25-44) and the $73 \%$ of sample was women, as shown in Table 1.

\section{IES-R data}

Mean of IES-R data is reported in Fig. 1. Thirty-three percent of the sample obtained a higher score (more than 33). As shown in Table 2, no analyzed characteristics (age, sex, professional role and working seniority) were statistically significant for post-traumatic stress symptoms $(p>0.5)$.

\section{ANAC data}

Mean values of ANAC questionnaire are reported in Fig. 1. For all items (A-P), the median score was high. Only age inferior to 30 years and lower working seniority (inferior to 5 years) were statistically significant for higher score for all items $(p<0.05)$.

\section{MBI data}

MBI data are reported in Table 2. In terms of EE, no one had a high score (superior of 30 ), while approximately $70 \%$ of sample had a lower score (inferior to 17). In regard to DP, $27 \%$ had a high score (superior of 12 ) and $65,4 \%$ a moderate score. The PA was high in $50 \%$ of cases (with a score inferior to 33) and low in $7.7 \%$ of sample. Only working seniority (inferior to 5 years) and male sex were correlated with high level of PA ( $p: 0.05 ; p: 0.03)$.

\section{Discussion}

All the healthcare workers are at increased risk of mental health difficulties, especially for oncological scenario in which all people are in contact with suffering and death $[14,15]$. Several data revealed that a considerable number of healthcare professionals working in oncology service showed burnout symptoms, allowing to identify the main 
Fig. 1 Mean value of IES-R and ANAC questionnaire

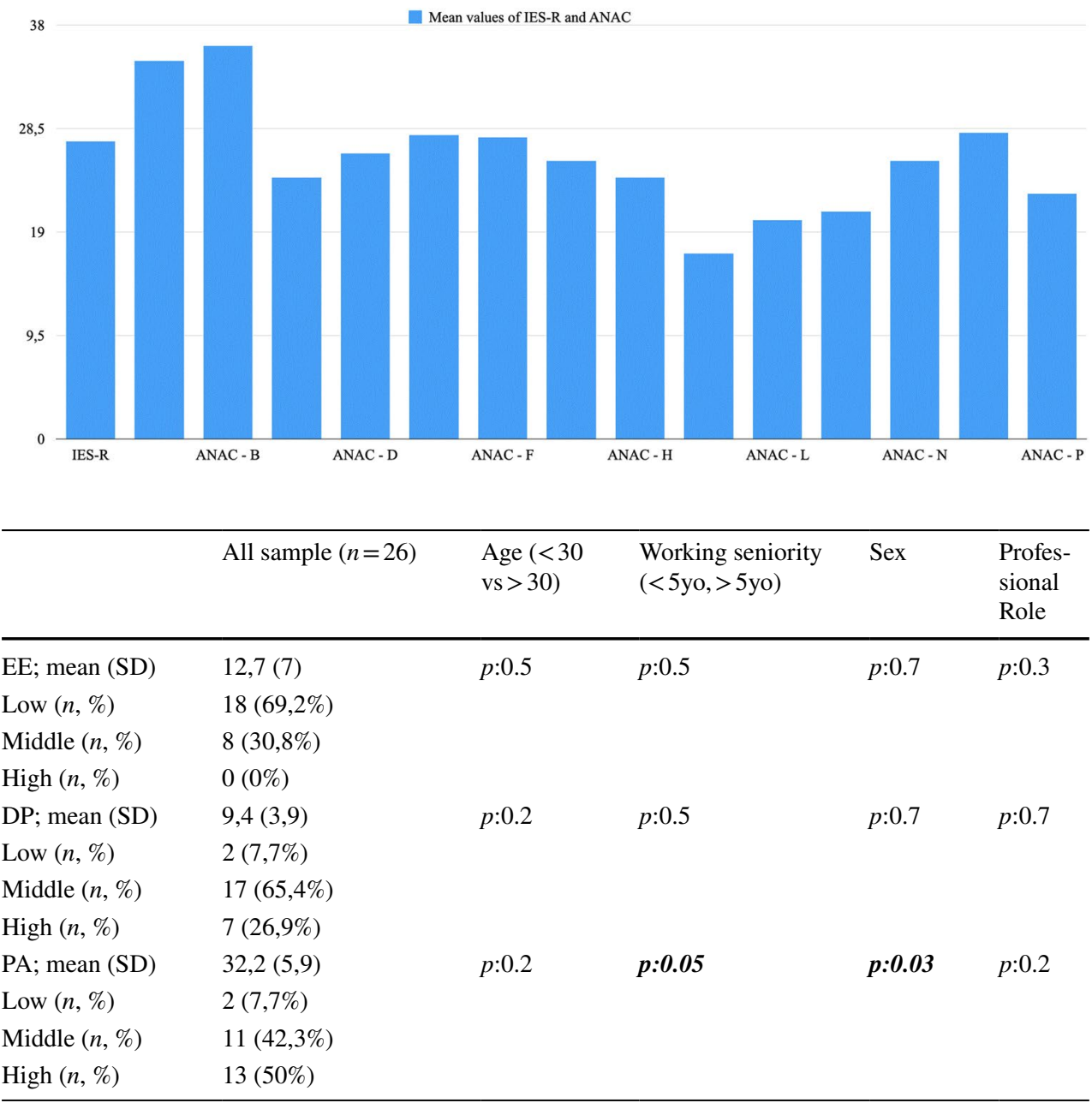

MBI, Maslach Burnout Inventory; EE, Emotional exhaustion; DP, Depersonalisation; PA, Personal accomplishment sources of work unsatisfaction: work overload, organizational problems, communication and emotional aspects with patients and colleagues [19].

During the present pandemic, all people experienced a negative impact on their daily life. A recent systematic review and meta-analysis, focused on stress and anxiety prevalence among the general population, showed a prevalence of stress in $29.6 \%$ of population (total sample size of 9074), a prevalence of anxiety in $31.9 \%$ (sample size of 63.439 ) and a prevalence of depression in $33.7 \%$ of population (sample size of 44.531 people) [3].

Healthcare workers suffered the weight of organizational changes in the daily work routine. In the radiotherapy scenario, the Italian Association of Radiotherapy and clinical Oncology (AIRO) produced a guidance document for all Italian Radiation Oncologists in order to try to homogenize the operational procedures of activities during the ongoing COVID-19 pandemic emergency for patients and healthcare professionals [20]. Patient-doctor interaction is an important factor affecting workflow especially in a radiation therapy facility. Nevertheless, this factor is often underrated; however, levels of satisfaction may influence compliance, continuity of treatments and patient-doctor communication improving the overall quality of clinical care [21].

The pandemic, the lockdown and the organizational health changes to deal with the pandemic could be causes of stress in health personnel in the oncology field; therefore, based on this background, the aim of the present self-survey-based analysis is to evaluate the psychological impact of COVID-19 in radiation oncology department workers, reporting data on burnout, post-traumatic stress symptoms and organizational well-being.

To our knowledge, this is the first study published about this issue.

The surveys get a "real-life snapshot" of current issues and stimulate discussion leading to the development of tailored interventions [22]

Regarding distress and psychological symptoms, in Chinese physicians and nurses, more than $70 \%$ reported symptoms of distress [6]. Similar data were reported for Oman 
physicians [4], French urologists [23] and general practitioners (GPs), for whom up to $42 \%$ of burnout symptoms and $11 \%$ post-traumatic stress symptoms were reported [11].

An Italian study of 132 GPs working in Genoa showed that $30 \%$ of people reported moderate/severe depression symptoms associated with anxiety and insomnia [9].

The present data reported a high level of post-traumatic stress symptoms: $33 \%$ of workers showed a score of IES-R over 33 . Any analyzed factors, such as age or sex, were associated with symptom onset. However, as the duration and the types of stressors can vary, also the responses can be significantly variable based on individuals' cognitive, emotional and neurobiological process, including the style of attachment or the copying which are activated as response to stress [13]. The differences in stress responses, among general population, contribute to psychological or physical symptoms with higher or lower rates and severity.

Nevertheless, among the seven cases with high IES-R, three workers lived alone away from family, two workers had parents with severe COVID-19 symptoms, and one worker had an oncological patient in her family.

Regarding Burnout syndrome, no one experienced high level of EE, while 27 and $50 \%$ of sample experienced high level of DP and PA, respectively. Only working seniority (inferior to 5 years) and male sex were correlated with high level of PA ( $p: 0.05 ; p: 0.03)$.

In terms of organizational well-being, the high score reported for each ITEM showed that the organizational changes during COVID-19 period were well tolerated due to the knowledge of their utility in terms of patients and personal security. Another analysis, despite without the use of an organizational well-being questionnaire, showed the same results; in fact, Francolini et al. showed that organizational changes during pandemic did not significantly affect normal radiotherapy workflow in selected situations [24].

Moreover, the higher score was correlated with lower age and working seniority, probably due to inferior experience in terms of working organization. The managers (including physicians) showed lower scores (albeit with high medians) compatible with greater responsibility (cause of stress and discontent) and a more complete view of the organization.

\section{Conclusion}

Despite the limitations of the study (sample size, no data before COVID-19), these data are interesting. Based on selfreport questionnaires, up to $33 \%$ reported post-traumatic symptom and up to 50\% experienced high level of burnout in terms of perception of poor competence. Surely, intervention to promote mental health well-being should be implemented in radiation oncology department.
Acknowledgements All radiotherapy staff: Gianluca Ladisa, Luciana Branà, Annarita Ciocia, Domenico Curci, Alberto Aga, Francesco Berloco, Marina Demasi, Federica Fragnoli, Rosilda Cuscito, Giuseppe Sanfrancesco, Christian Depascali, Chiara Inellicati, Rosalinda Parabita, Daniela Nardelli, Maria Nardiello, Valeria De Candia.

Authors' contributions $\mathrm{MC}$ and $\mathrm{AF}$ and $\mathrm{SC}$ defined the study and analyzed data; MC, FG, AS, RC, MPC, IB, SC and AF wrote the paper.

Funding None.

\section{Declarations}

Conflicts of interest The authors declared no conflicts of interest.

Ethical approval This article does not contain any studies with human participants or animals performed by any of the authors.

Consent to participate Informed consent was signed.

\section{References}

1. Zhu N, Zhang D, Wang W et al (2020) A novel coronavirus from patients with pneumonia in China, 2019. N Engl J Med 382:727733. https://doi.org/10.1056/NEJMoa200101

2. Miaskowski C, Paul SM, Snowberg K et al (2020) Stress and symptom burden in oncology patients during the COVID-19 pandemic. J Pain Symptom Manage 60(5):e25-e34. https://doi.org/ 10.1016/j.jpainsymman.2020.08.037

3. Salari N, Hosseinian-Far A, Jalali R et al (2020) Prevalence of stress, anxiety, depression among the general population during the COVID-19 pandemic: a systematic review and metaanalysis. Global Health 16(1):57. https://doi.org/10.1186/ s12992-020-00589-w

4. Badahdah AM, Khamis F, Mahyijari NA (2020) The psychological well-being of physicians during COVID-19 outbreak in Oman. Psychiatry Res 289:113053. https://doi.org/10.1016/j.psychres. 2020.113053

5. Du J, Dong L, Wang T et al (2020) Psychological symptoms among frontline healthcare workers during COVID-19 outbreak in Wuhan. Gen Hosp Psychiatry 67:144-145. https://doi.org/10. 1016/j.genhosppsych.2020.03.011

6. Lai J, Ma S, Wang Y et al (2020) Factors associated with mental health outcomes among health care workers exposed to coronavirus disease 2019. JAMA Netw Open 3:e203976. https://doi.org/ 10.1001/jamanetworkopen.2020.3976

7. KangL MaS, ChenM, et al (2020) Impact on mental health and perceptions of psychological care among medical and nursing staff in Wuhan during the 2019 novel coronavirus disease outbreak: a cross-sectional study. Brain Behav Immun 87:11-17. https://doi. org/10.1016/j.bbi.2020.03.028

8. Zhang C, Yang L, Liu S et al (2020) Survey of insomnia and related social psychological factors among medical staff involved in the 2019 novel coronavirus disease outbreak. Front Psychiatr 11:306. https://doi.org/10.3389/fpsyt.2020.00306

9. Amerio A, Bianchi D, Santi F et al (2020) Covid-19 pandemic impact on mental health: a web-based cross-sectional survey on a sample of Italian general practitioners. Acta biomed 91:83-88. https://doi.org/10.23750/abm.v91i2.9619

10. El-Hage W, Hingray C, Lemogne C et al (2020) Health professionals facing the coronavirus disease 2019 (COVID-19) pandemic: 
what are the mental health risks? Encephale 46:S73-S80. https:// doi.org/10.1016/j.encep.2020.04.008

11. Lange M, Joo S, Couette PA et al (2021) Impact on mental health of the COVID-19 outbreak among general practitioners during the sanitary lockdown period. Ir J Med Sci. https://doi.org/10.1007/ s11845-021-02513-6

12. Lange M, Joo S, Couette PA et al (2020) Impact on mental health of the COVID-19 outbreak among community pharmacists during the sanitary lockdown period. Ann Pharm Fr 78(6):459-463. https://doi.org/10.1016/j.pharma.2020.09.002

13. Gregucci F, Caliandro M, Surgo A et al (2020) Cancer patients in Covid-19 era: swimming against the tide. Radiother Oncol 149:109-110. https://doi.org/10.1016/j.radonc.2020.04.002

14. Guerra J, Patrício M (2019) Burnout in radiation therapists: systematic review with meta-analysis. Eur J Cancer Care (Engl) 28(3):e12938. https://doi.org/10.1111/ecc.12938

15. Ciammella P, De Bar B, Fiorentino A et al (2013) The "BUONGIORNO" project: burnout syndrome among young Italian radiation oncologists. Cancer Invest 31(8):522-528. https://doi.org/10. 3109/07357907.2013.830735

16. Creamer M, Bell R, Failla S (2003) Psychometric properties of the impact of event scale - revised. Behav Res Ther 41:1489-1496. https://doi.org/10.1016/j.brat.2003.07.010

17. Jackson SE, Leiter MP, Maslach C (1996) MBI: Maslach burnout inventory. Consulting Psychologists Press, Palo Alto, CA

18. https://www.anticorruzione.it/portal/rest/jcr/repository/collaborat ion/Digital\%20Assets/anacdocs/Attivita/ArchivioStorico/Benes
sereOrganizzativo/ModelliDiIndagine/Allegato-A-Indagini-supersonale-dipendente2.pdf

19. Font A, Corti V, Berger R (2015) Burnout in healthcare professionals in oncology. Proc Econ Financ 23:228-232

20. https://www.radioterapiaitalia.it/wp-content/uploads/2020/04/ LATEST_ENG_AIRO-COVID-19.pdf

21. Desideri I, Francolini G, Ciccone LP et al (2021) Impact of COVID-19 on patient-doctor interaction in a complex radiation therapy facility. Support Care Cancer 29(6):2931-2937

22. Gregucci F, Fozza A, Falivene S et al (2020) Present clinical practice of breast cancer radiotherapy in Italy: a nationwide survey by the Italian Society of Radiotherapy and Clinical Oncology (AIRO) Breast Group. Radiol Med 125(7):674-682

23. Abdessater M, Rouprêt M, Misrai V et al (2020) COVID19 pandemic impacts on anxiety of French urologist in training: outcomes from a national survey. Prog Urol 30(8-9):448-455. https:// doi.org/10.1016/j.purol.2020.04.015

24. Francolini G, Desideri I, Stocchi G et al (2021) Impact of COVID19 on workload burden of a complex radiotherapy facility. Radiol Med 126(5):717-721

Publisher's Note Springer Nature remains neutral with regard to jurisdictional claims in published maps and institutional affiliations. 